\title{
Modelo de pronóstico para estimar el comportamiento del precio en bolsa de la energía en Colombia
}

\author{
Lucero Gómez-Cano ${ }^{1}$ \\ Sandra Catalina-Cuellar² \\ Raphael Méndez-Vargas ${ }^{3}$
}

Fecha de recepción: 14 de octubre de 2020

Fecha de aprobación: 23 de diciembre de 2020

\section{Resumen}

El objetivo de este trabajo es proponer un modelo estadístico que permita pronosticar el precio de la energía en bolsa en Colombia, incorporando el efecto de algunas de las variables que mayor impacto tienen sobre la formación de este. Para realizar el análisis, se procede con una contextualización del funcionamiento del mercado eléctrico en Colombia, dado que su estructura y modelo de operación determinan la formación de los precios de mercado, entre los cuales, el precio de energía en bolsa se convierte en uno de los que registra mayor volatilidad. Para identificar el modelo de pronóstico se utiliza la metodología de Box-Jenkins de series de tiempo y se propone el mejor modelo SARIMA, SARIMAX y VAR, a partir de los cuales se realiza los pronósticos correspondientes y los análisis de resultados.

Fundación Universitaria Los Libertadores (Bogotá-Distrito Capital, Colombia). lgomezc01@libertadores.edu.co. ORCID: 0000-0002-0349-6633

2 Fundación Universitaria Los Libertadores (Bogotá-Distrito Capital, Colombia). ccuellars@libertadores.edu.co. ORCID: 0000-0002-6735-8846

3 Fundación Universitaria Los Libertadores (Bogotá-Distrito Capital, Colombia). rrmendezv@libertadores.edu.co. ORCID: 0000-0003-3657-0059 
Palabras clave: mercado eléctrico colombiano; modelos SARIMAX; modelos VAR; precio energía en bolsa.

\section{Forecast Model to Estimate Energy Stock Price in Colombia}

\section{Abstract}

The aim of this work is to propose a statistical model to forecast the price spot of energy on the stock electric market in Colombia, incorporating the effect of some variables that have impact on its formation. To do it, we proceed with a contextualization of the electricity market in Colombia, because its structure and operating model determine the formation of market prices, among which the price spot of energy has the highest volatility. To identify the forecasting model, the BoxJenkins methodology of time series is used and the best SARIMA, SARIMAX and VAR model is proposed, from which the corresponding forecasts and analysis of results are made.

Keywords: colombian electric market; price spot of energy; SARIMAX model; VAR model.

\section{Para citar este artículo:}

Gómez-Cano, L., Catalina-Cuellar, S., Méndez-Vargas, R. (2021). Modelo de pronóstico para estimar el comportamiento del precio en bolsa de la energía en Colombia. Pensamiento y Acción, 30, 69-90.

Esta obra está bajo licencia internacional Creative Commons Reconocimiento 4.0

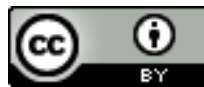




\section{Introducción}

El mercado eléctrico colombiano ha sufrido grandes transformaciones a partir de la liberalización dispuesta por las leyes de servicios públicos (República de Colombia, 1994a) y del mercado eléctrico (República de Colombia, 1994b), con las cuales se creó el mercado de energía mayorista, y con él, la introducción de la competencia en las actividades de generación y comercialización, la estipulación de la desintegración vertical entre la generación y el transporte; e implementación de dos mercados para la prestación del servicio a los consumidores, el de los usuarios regulados y el de los no regulados (Rendon, Hiniestrosa, y Moreno, 2011).

Dentro de los cambios presentados, también se encuentra la definición de dos formas de transar la energía entre los agentes generadores y los agentes comercializadores de energía con el fin de abastecer la demanda de los usuarios; la primera a través de los contratos de largo plazo, donde se fijan cantidades, precios y tiempo de entrega, y la segunda a través de un mercado de corto plazo o spot que se forma a través de una subasta confidencial de los agentes generadores, la cual recoge el impacto de factores internos, externos y de mercado (Sabogal, 2016).

Las ventas de energía en bolsa, las cuales corresponden al segundo mecanismo expresado anteriormente, representan un promedio del $23 \%$ de participación en las ventas totales de energía en los últimos 20 años en Colombia, según cifras del operador del mercado XM (XM, 2020). Este hecho, implica una necesidad de comprender los aspectos fundamentales que intervienen en la formación del precio asociado, pues a diferencia de la venta de energía a través de contratos, la cual responde a una negociación o licitación entre las partes, en la bolsa existen múltiples factores que inciden directa o indirectamente en la formación del precio de la energía que se va a transar a través de este mecanismo.

Este hecho explica que la estimación de los precios sea un reto para los diferentes agentes que participan en el mercado, desde los generadores y comercializadores, hasta los clientes finales, gobierno e instancias de control o regulatorias. En este sentido, realizar el pronóstico del precio de energía en bolsa es importante, entre otros, para identificar la evolución futura del precio, estimar en 
qué medida es necesario estimular la expansión de la capacidad instalada que requiere el país para aumentar la eficiencia del mercado, y finalmente proveer señales de mercado sobre el retorno que obtendrían los inversionistas al emprender proyectos de generación (Barrientos y Toro, 2017).

Adicionalmente, este precio es insumo fundamental para la definición de las estrategias comerciales de los agentes de mercado, a partir de las cuales se establecen las cantidades de energía que se negociarán a través de contratos de largo plazo y a través del mercado spot, de manera que se optimice el beneficio neto de cada una de las partes en el periodo proyectado. De lado del gobierno y los entes reguladores, conocer la proyección del precio de bolsa permite identificar posibles anomalías de mercado e intervenciones que deberían realizarse para asegurar un control del poder de mercado de las empresas oligopólicas (Santamaría et al., 2009).

En este contexto, el pronóstico de una serie de precios de energía es una tarea difícil y compleja, debido a que en ella se presentan diferentes niveles de estacionalidad y se observa la influencia de las múltiples variables exógenas de tipo cualitativo y cuantitativo que fueron descritas anteriormente. Para lograrlo, es necesario realizar una revisión de las principales metodologías de análisis multivariado de series de tiempo o regresión, de manera que se pueda proponer un modelo de pronóstico que, además de cumplir con los criterios estadísticos, permita realizar una proyección que se acerque a la realidad.

\section{Referentes Teóricos}

\section{Contextualización del mercado eléctrico en Colombia}

El sector eléctrico colombiano ha sido pieza fundamental en el desarrollo económico del país $y$, a pesar de que ha sufrido grandes transformaciones desde sus inicios en los albores del siglo XX, fue con la introducción de la Constitución Política de Colombia del año 1991 donde se dieron las condiciones para abrir el mercado a la competencia privada y para consolidar el papel del Estado como ente diseñador de 
políticas, regulador y controlador, que busca un desempeño eficiente del sector (Alvarez y Tamayo, 2006).

En este contexto, el mercado eléctrico colombiano cambió radicalmente con la implementación de la ley de servicios públicos (República de Colombia, 1994a) y la ley eléctrica (República de Colombia, 1994b), las cuales se fundamentan en el hecho de que las empresas comercializadoras y los grandes consumidores adquieran la energía y potencia en un mercado de grandes bloques de energía, que opera libremente de acuerdo con las condiciones de oferta y demanda. (Bello y Beltrán, 2010).

Los agentes que participan en este sector son los siguientes: agentes generadores quienes se encargan de la producción de energía eléctrica, los agentes transportadores quienes no compran ni venden energía, sino que la movilizan en todo el sistema interconectado, los agentes distribuidores quienes se encargan de recibir la energía que los transportadores despachan para su consumo por el usuario final y los agentes comercializadores quienes desempeñan la labor de intermediación entre los usuarios finales de la energía y los que la generan, transportan y distribuyen. En la Fig. 1 se observan los participantes en el mercado y su interacción con XM, empresa encargada de la operación del sistema.

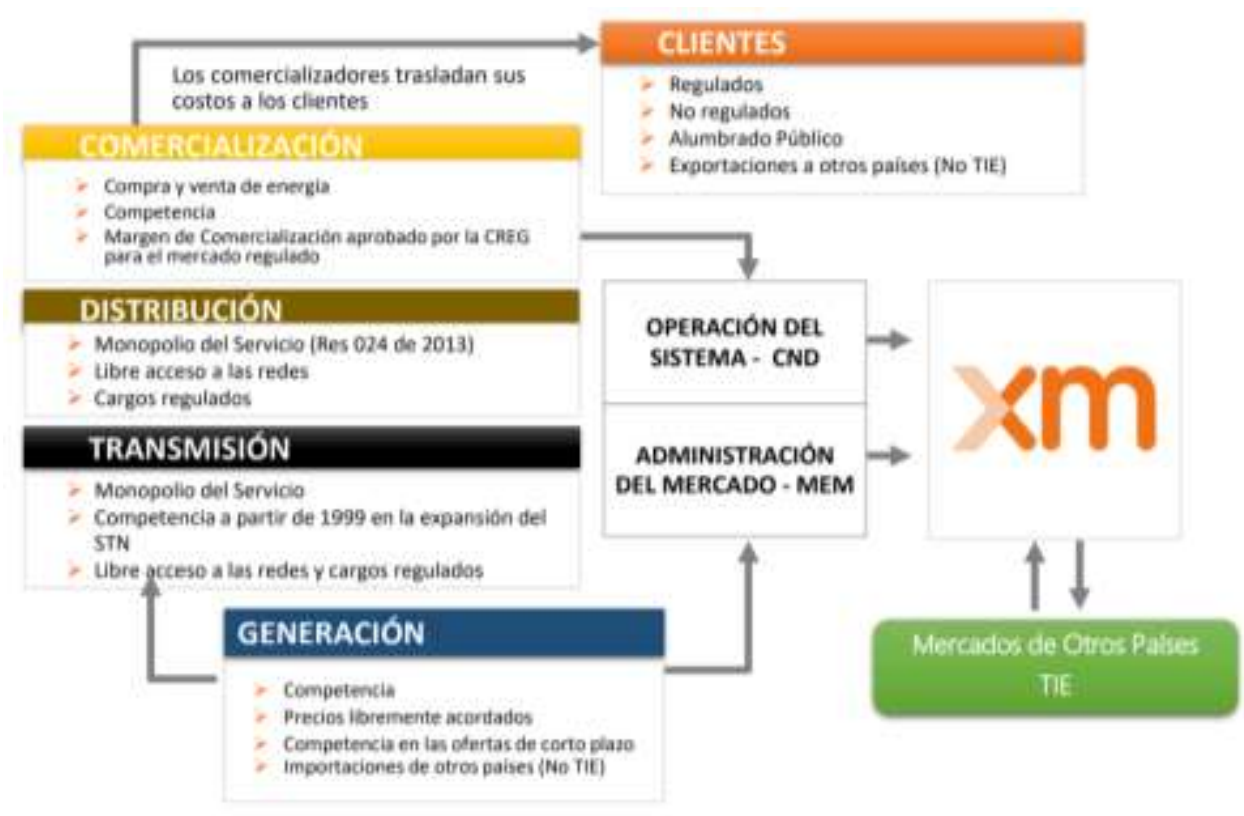

Fig. 1. Estructura del sector eléctrico (XM, 2019). 
El funcionamiento del Mercado de Energía Mayorista (MEM) está soportado en una bolsa de energía o pool de generadores, donde se realizan los intercambios comerciales definidos en el contexto de un mercado con resolución horaria y soportado por un operador del sistema interconectado nacional (Gil y Maya, 2008). Existen dos mecanismos para transar la energía en el MEM, el primero relacionado con contratos de largo plazo donde se establecen con anterioridad el precio y las cantidades de energía a entregar, y el segundo, relacionado con un mercado de corto plazo que atiende los requerimientos de energía cuando son mayores a los pactados en los contratos bilaterales y el comercializador deberá contratar el excedente de energía a través del precio de bolsa (Martın y Pineros, 2020).

Desde el punto de vista de la matriz de generación, Colombia registra una alta dependencia de los recursos hídricos, con una capacidad instalada en centrales hidráulicas de alrededor de un $70 \%$ del total; esta situación se repite con la generación real, la cual depende de los efectos climáticos variables que implican una disminución o aumento de la oferta de generación hidráulica (Ver Fig. 2 yFig. 3).

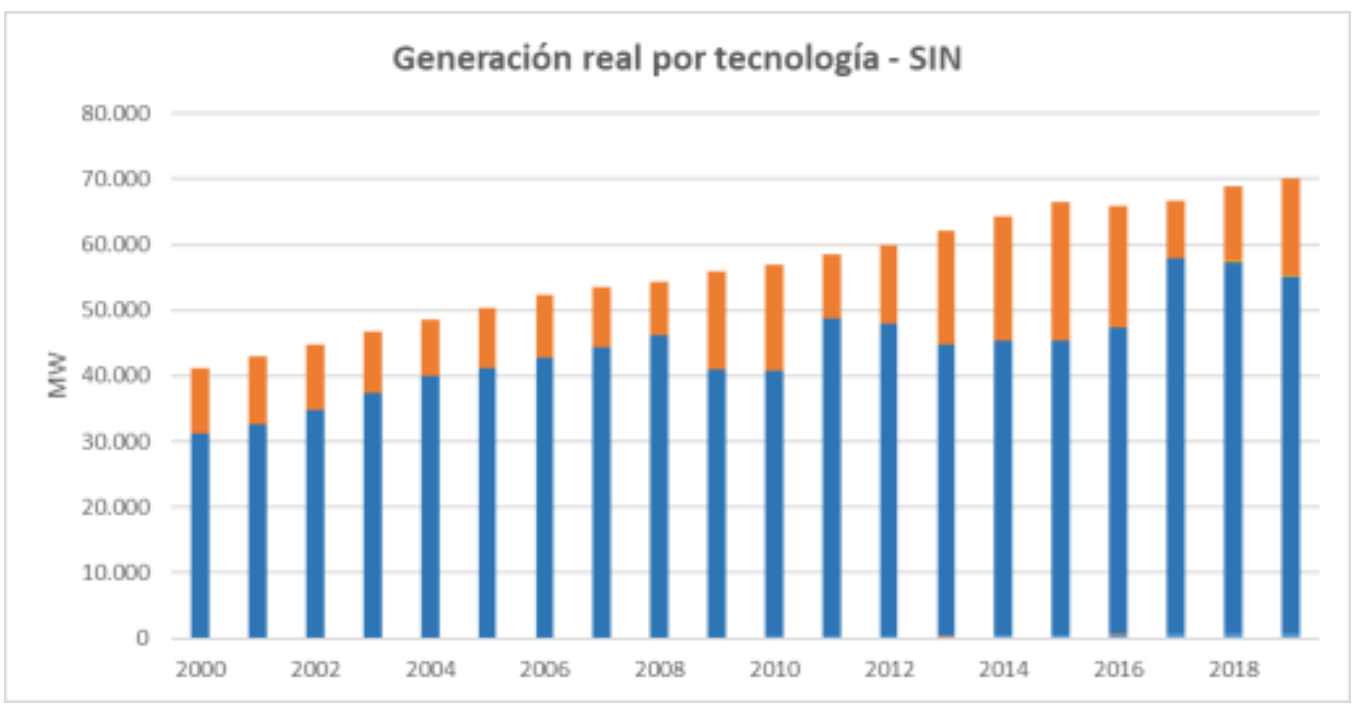

Fig. 2. Evolución de la composición de la generación real por tecnología en el Sistema Interconectado Nacional - SIN. Elaboración propia con base en (XM, 2020). 


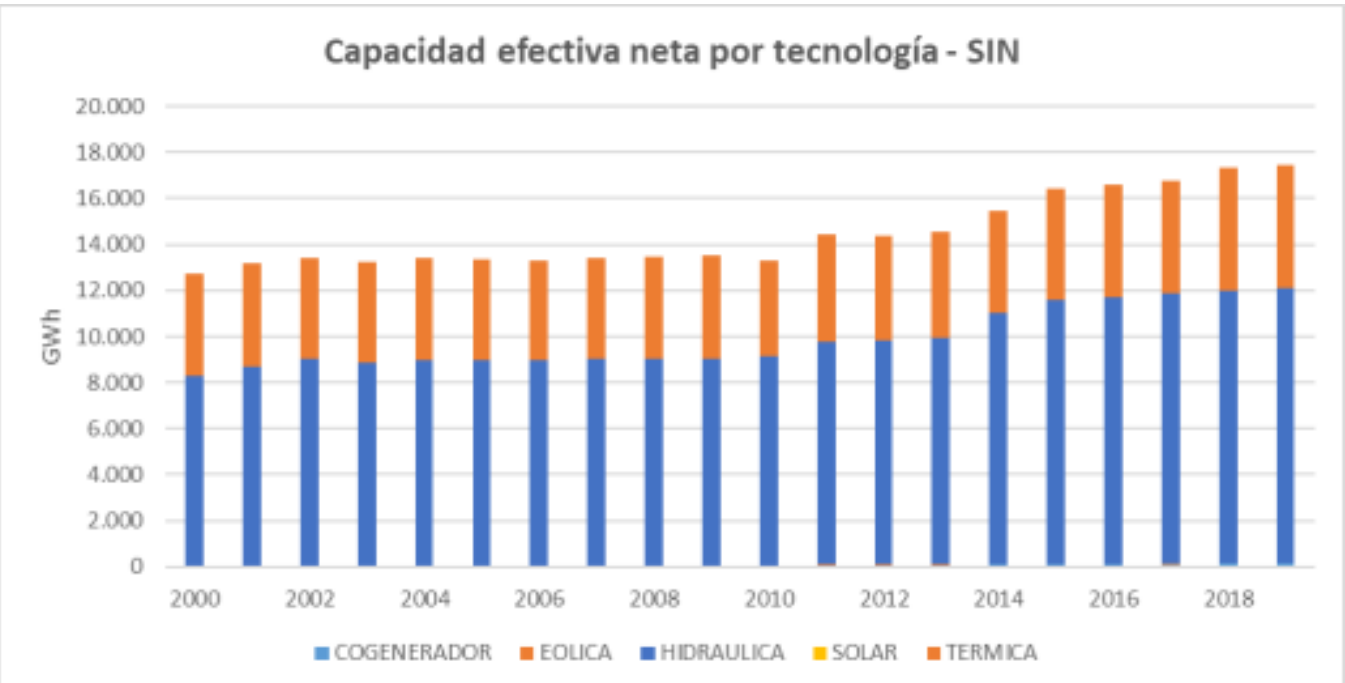

Fig. 3. Evolución de la composición de la capacidad efectiva neta tecnología en el Sistema Interconectado Nacional - SIN. Elaboración propia con base en (XM, 2020).

\section{Formación de los precios de bolsa (precio spot)}

A diferencia de los contratos de largo plazo, donde los precios son establecidos de manera previa a la entrega de la energía, en la bolsa de energía los precios se forman de acuerdo con el mecanismo de oferta y demanda definido por el mercado. El procedimiento consiste en una subasta a sobre cerrado, donde los generadores deben presentar al Centro Nacional de Despacho una oferta por central y periodicidad diaria, un precio único para la cantidad que están dispuestos a vender para hora. Dicho procedimiento se realiza con el fin de garantizar la libre competencia y reflejar las condiciones del mercado (Galindo, 2017).

Con esta información, el operador del mercado ordena las ofertas de menor a mayor precio hasta el último oferente con el cual se pueda satisfacer la demanda esperada (despacho ideal). Posteriormente, se van otorgando méritos de despacho a cada una de las plantas y unidades de acuerdo con los precios ofertados el día anterior, hasta que la asignación iguale la demanda real del mercado para cada hora del día, sin considerar las restricciones, regulación secundaria de frecuencia ni pérdidas. En ese punto se dará el cierre de precios con el que serán remunerados los agentes con mérito el día anterior a través del método de subasta de inversa (Rojas, 2016). Una representación de este mecanismo se ilustra en la Fig. 4. 


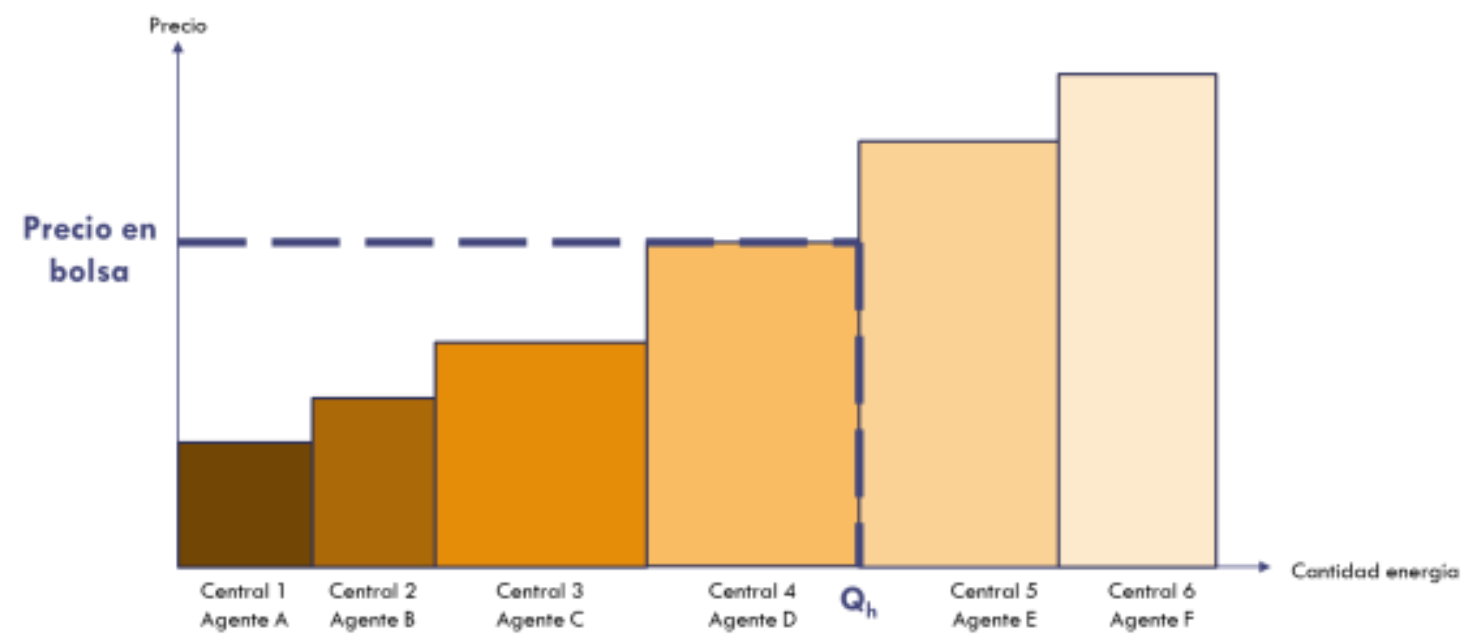

Fig. 4. Ejemplo de subasta inversa en la hora $h$ con una demanda $Q_{h}$. (Hurtado et al., 2014).

\section{Variables que inciden en el precio de bolsa}

A pesar de que el precio de bolsa de energía es una variable exclusiva del sector eléctrico, la cual, en principio pareciera influenciada solo por condiciones de oferta y demanda, en la realidad, esta se ve impactada por variables del mismo sector y de otros sectores económicos, que le pueden provocar movimientos positivos (crecimiento del precio) o negativos (disminución del precio).

Por un lado, se encuentran variables como el PIB, el precio del gas, la disponibilidad de generación, la demanda máxima de potencia, las exportaciones a Ecuador y la capacidad efectiva neta del sistema, las cuales tienen una correlación positiva con el precio de bolsa, esto es, ante incrementos en cada una de estas variables de manera individual, se presentan mayores precios de bolsa. Por otro lado, la hidrología, la demanda esperada del SIN, el nivel de los embalses, el precio de escasez y el margen de reserva tienen una correlación negativa con el precio de bolsa, lo que implica que incrementos en el nivel de estas variables ocasionan caídas en el precio de bolsa. (Santamaría et al., 2009)

Sin embargo, de las anteriores variables hay algunas que tienen mayor impacto que otras, dependiendo de su naturaleza e influencia sobre la oferta y demanda de la energía. Por ejemplo, a causa del gran componente hidráulico, en Colombia existe una alta vulnerabilidad a eventos secos y de difícil predicción, como el ENSO (El Niño Southern Oscilation), por lo cual es importante el estudio de los 
aportes hídricos como predictor relevante del comportamiento del precio de energía en bolsa (Barrientos y Toro, 2016).

Otra variable destacable es el comportamiento de la demanda de energía, la cual, en general, es bastante inflexible debido a que la energía eléctrica no es acumulable una vez generada, y, por ende, la producción y el consumo deben ser iguales en todo momento. Así mismo, los usuarios por lo general consumen dependiendo de sus necesidades diarias sin tener en cuenta la variación de los precios de energía (Díez, 2015). En este sentido, un aumento en la demanda logra impactar los precios de la energía en bolsa, en la medida que la oferta de energía tenga más limitaciones para atenderla, dado que, por ejemplo, durante el tiempo seco es necesario acudir a la generación térmica para dar respuesta a las necesidades establecidas por la demanda y tiene un costo variable significativamente mayor al costo de la generación hidráulica.

En la Fig. 5 se puede observar el comportamiento del precio de energía en bolsa mensual entre los años 2000 y 2019. En este periodo se registraron incrementos importantes en el precio, los cuales, en su mayoría coincidieron con el desarrollo del fenómeno El Niño o disminución de los aportes hídricos.

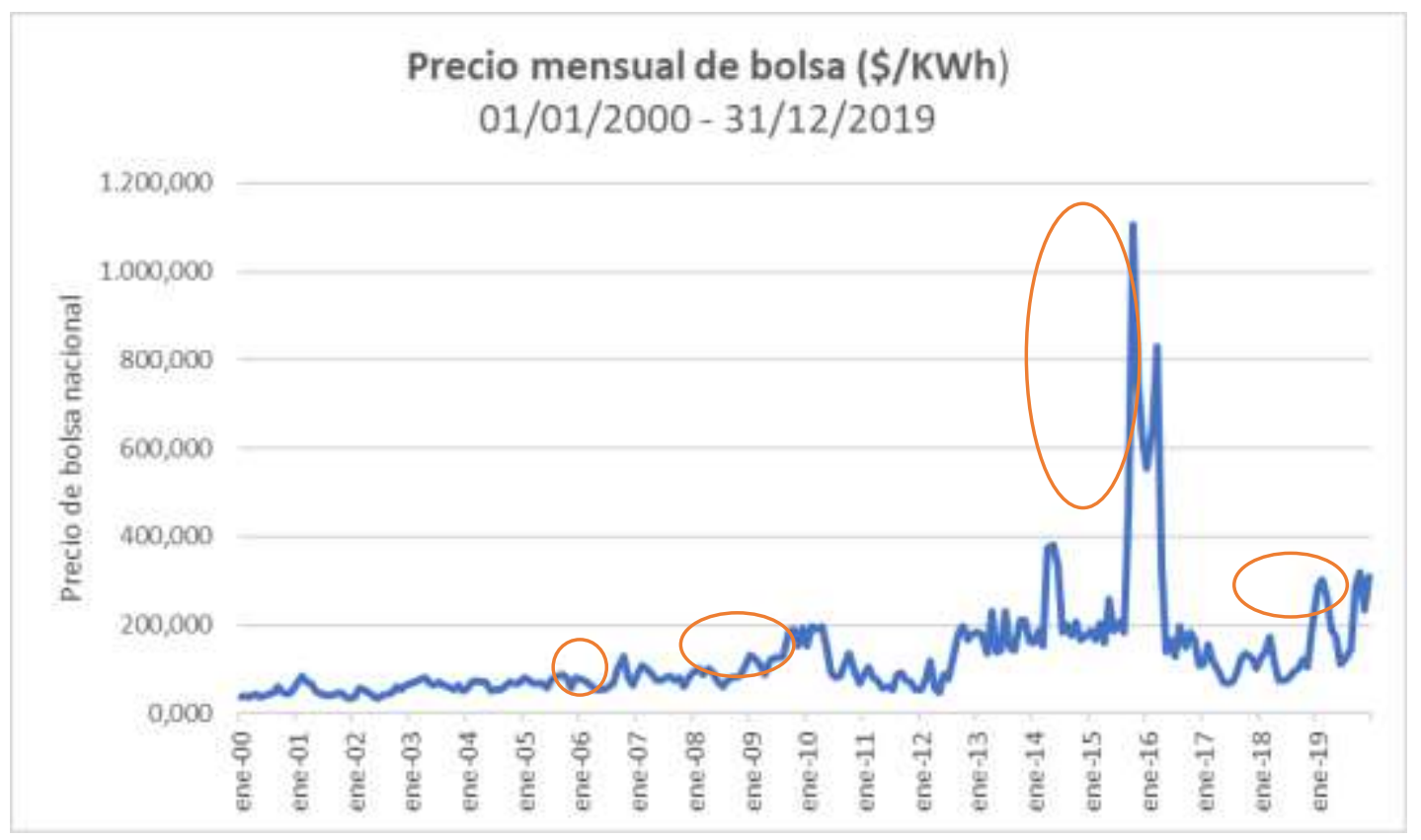

Fig. 5. Evolución precio mensual de la bolsa de energía en Colombia. Elaboración propia a partir de (XM, 2020). 


\section{Metodología}

En el presente trabajo se utiliza la metodología Box-Jenkins para generar un modelo que permita pronosticar el precio de bolsa, a partir de la aplicación de diferentes modelos de series de tiempo: SARIMA, SARIMAX y VAR.

El área de estudio comprende el mercado de energía eléctrica de Colombia, dentro del cual se profundiza en el precio de energía en bolsa y se presentan variables relevantes como la demanda de energía, los aportes hídricos, el volumen útil de los embalses y la disponibilidad real de generación de las centrales del Sistema Interconectado Nacional.

El universo del presente trabajo corresponde a los datos mensuales del precio de bolsa y de las variables que complementan el análisis, presentados desde la creación del Mercado de Energía Mayorista en 1995. La muestra comprende la información entre enero de 2000 y diciembre de 2019, la cual representa el 83\% de la información total hasta 2019; entre otras, se utiliza esta muestra debido a que XM (empresa encargada de operar el sistema y administrar la información correspondiente) tiene datos publicados a partir del año 2000 y la información del 2020 se utilizará como datos de entrenamiento de los modelos analizados.

\section{Resultados}

\section{Análisis descriptivo de las series}

En la Fig. 6 se observa el comportamiento de la serie de precios de bolsa entre el año 2000 y diciembre de 2019. Los principales incrementos se relacionan directamente con la presentación del Fenómeno El Niño en el país, con especial énfasis en los que ocurrieron en los años 2009-2010 y 2015-2016. Por el contrario, los descensos del precio de bolsa están relacionados con el Fenómeno La Niña o aumentos en los aportes hídricos, por ejemplo, en los años 2011 y 2017. 
Precios de bolsa

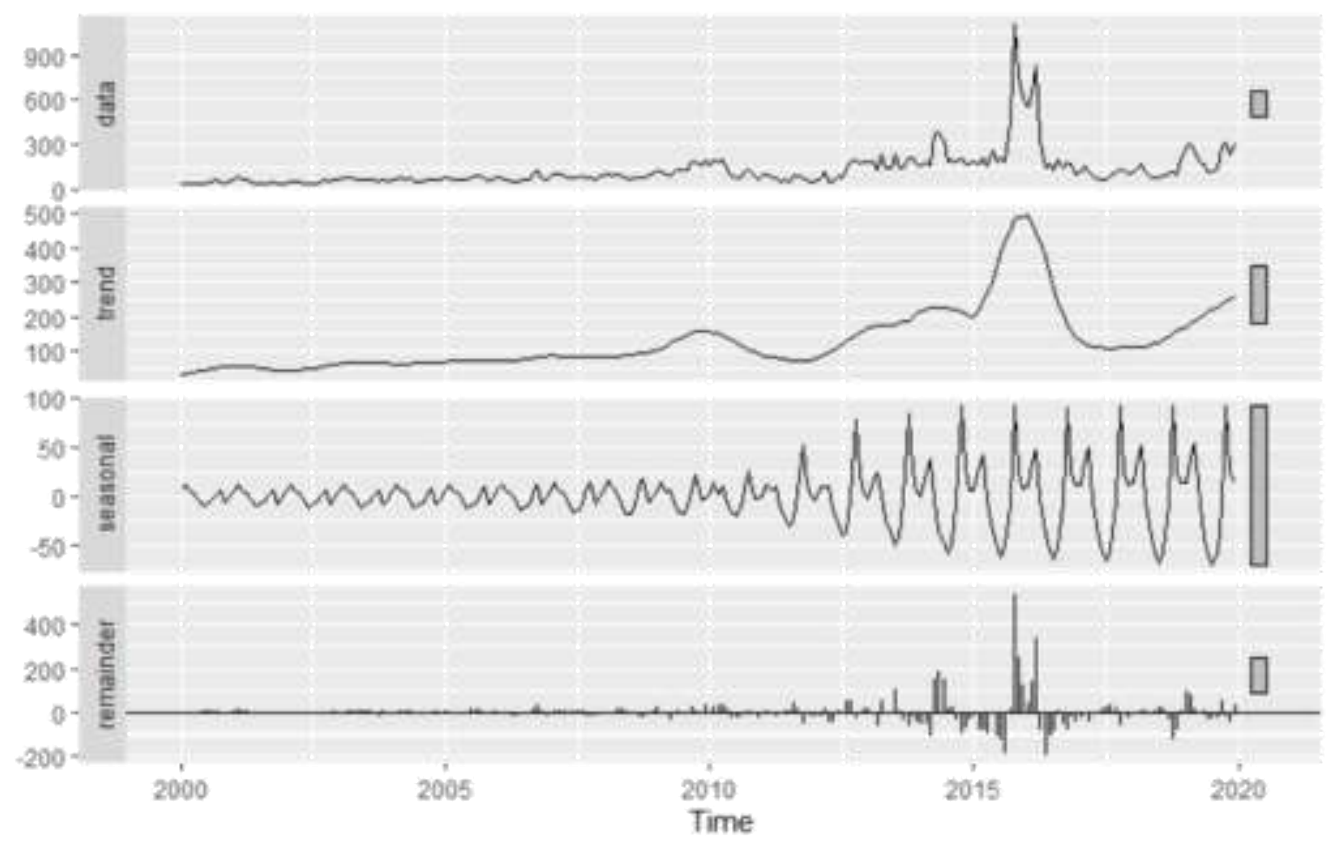

Fig. 6. Descomposición serie Precios de Bolsa.

En la descomposición de la serie también se pueden identificar ciclos con dos picos en el año, el primero presentado en los meses de enero a marzo, cuando se presenta la estación de verano en el país, mientras que el segundo, con menor intensidad, entre los meses junio a agosto. El componente aleatorio ha ido creciendo, en la medida que la presentación de los fenómenos oscilatorios sobre el Océano Pacífico es más frecuentes e impredecibles.

En cuanto a las demás variables mostradas en la Fig. 7, en todas se pueden observar componentes cíclicos, mientras que el componente de tendencia es más claro en las series de demanda comercial y disponibilidad del parque generador en las cuales se explica el comportamiento creciente debido a la mayor demanda comercial derivada del crecimiento económico y a entrada de nuevas centrales de generación al parque generador de Colombia. 
Aportes hídricos

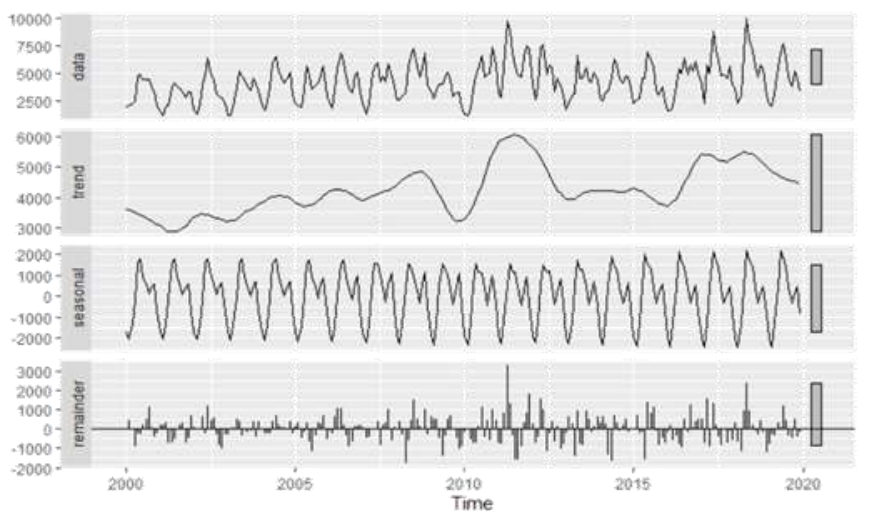

Volumen útil

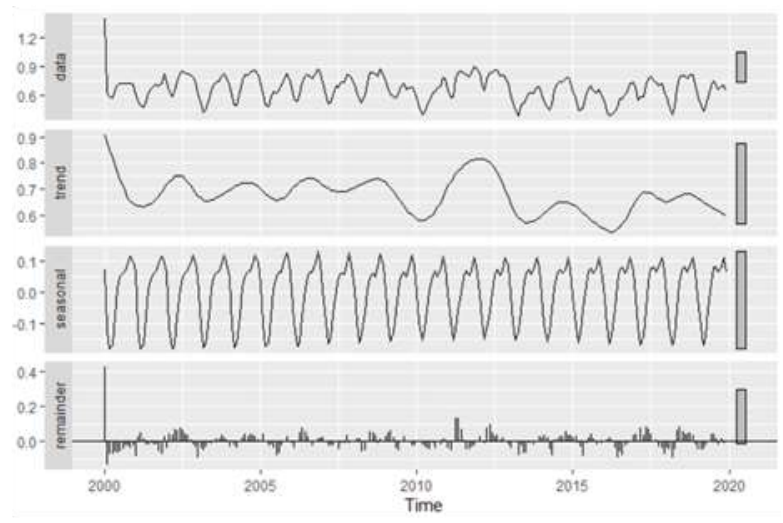

Demanda comercial

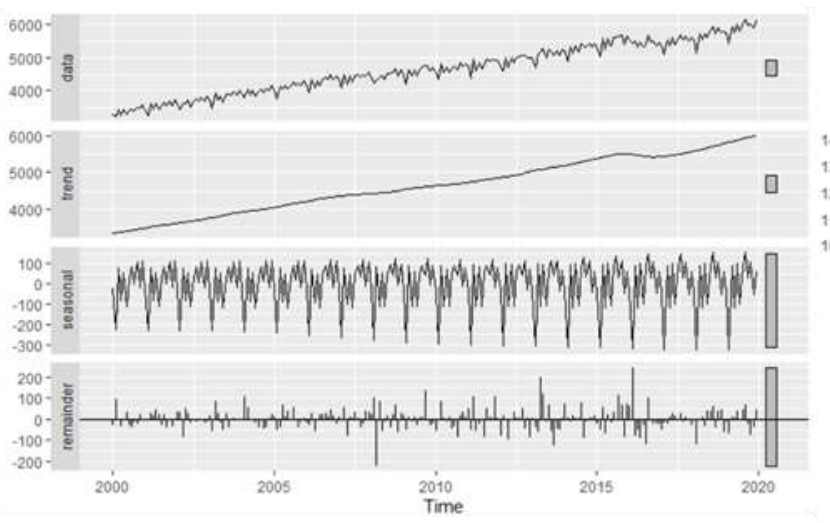

Disponibilidad parque generador

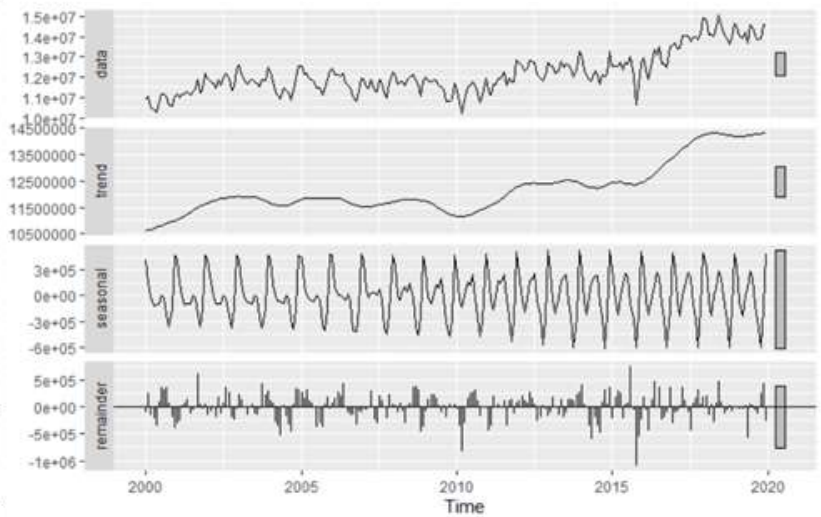

Fig. 7. Descomposición series aportes hídricos, volumen útil, demanda comercial y disponibilidad parque generador.

\section{Estacionariedad de las series}

Como condición previa para la identificación de modelos estadísticos según la metodología Box-Jenkins, es necesario evaluar la estacionariedad de las series a utilizar, es decir, que su comportamiento presente una media y varianzas constantes. En el caso del presente proyecto, se puede observar que todas las series pasan las pruebas de estacionalidad. Ver Tabla 1.

Tabla 1. Pruebas de estacionariedad de las series de tiempo.

\begin{tabular}{|l|r|r|r|l|}
\hline \multicolumn{1}{|c|}{ Serie } & $\begin{array}{c}\text { Augmented } \\
\text { Dickey-Fuller Test }\end{array}$ & $\begin{array}{c}\text { Dickey-Fuller Test } \\
\text { Unit Root Test }\end{array}$ & $\begin{array}{c}\text { Phillips-Perron } \\
\text { Unit Root Test }\end{array}$ & Resultado \\
\hline Bolsa & 0.01 & -5.5634 & 0.01 & Estacionaria \\
\hline Aportes & 0.01 & -9.2803 & 0.01 & Estacionaria \\
\hline Volumen útil & 0.01 & -7.7098 & 0.01 & Estacionaria \\
\hline
\end{tabular}




\begin{tabular}{|c|r|r|r|l|}
\hline Serie & $\begin{array}{c}\text { Augmented } \\
\text { Dickey-Fuller Test }\end{array}$ & $\begin{array}{c}\text { Dickey-Fuller Test } \\
\text { Unit Root Test }\end{array}$ & $\begin{array}{c}\text { Phillips-Perron } \\
\text { Unit Root Test }\end{array}$ & Resultado \\
\hline Demanda real & 0.01 & -7.5313 & 0.01 & Estacionaria \\
\hline Disponibilidad & 0.59 & -5.2232 & 0.01 & Estacionaria \\
\hline
\end{tabular}

\section{Evaluación y pronóstico modelo SARIMA}

Bajo el análisis univariado se evaluaron 6 modelos, de los cuales el modelo elegido es el número 1, debido a que pasa las pruebas de autocorrelación, normalidad y heterocedasticidad, además tiene el menor BIC.

Tabla 2. Pruebas Modelo SARIMA.

\begin{tabular}{|c|c|c|r|r|r|}
\hline Nombre & Descripción & \multicolumn{1}{c|}{ BIC } & Box.test & Jarque.Bera & Runs.test \\
\hline Modelo 1 & SARIMA $(0,1,18)(0,1,1)[12]$ & -1186.564 & 0.9368 & 0.1320 & 0.712 \\
\hline
\end{tabular}

Dado que el modelo no tiene explicación en los compontes autorregresivos ordinario y estacional, la ecuación está representada de la siguiente forma:

$\nabla_{s}^{D} \nabla^{d} x_{t}=\Theta_{Q}\left(B^{s}\right) \theta_{q}(B) w_{t}$ donde

$\nabla_{s}^{D}=\left(1+B^{12}\right)$

$\nabla^{d}=(1+B)$

$\theta_{q}=\left(1-0,1943 w_{t-1}-0,1045 w_{t-2}-0,1983 w_{t-8}-0,1618 w_{t-15}-0.1252 w_{t-18}\right.$

$\Theta_{Q}=\left(1-w_{t-12}\right)$

$x_{t}=$ Precio de bolsa en el periodo $\mathrm{t}$

$w_{t}=$ Ruido blanco en el periodo $t$

El Modelo 1 parece acomodarse parcialmente a la serie de tiempo, con excepción del pico presentado en el año 2015, aunque sí registra el incremento del 2016. Llama la atención la banda de confianza del pronóstico, pues se observa amplia para el periodo evaluada (Fig. 8). 


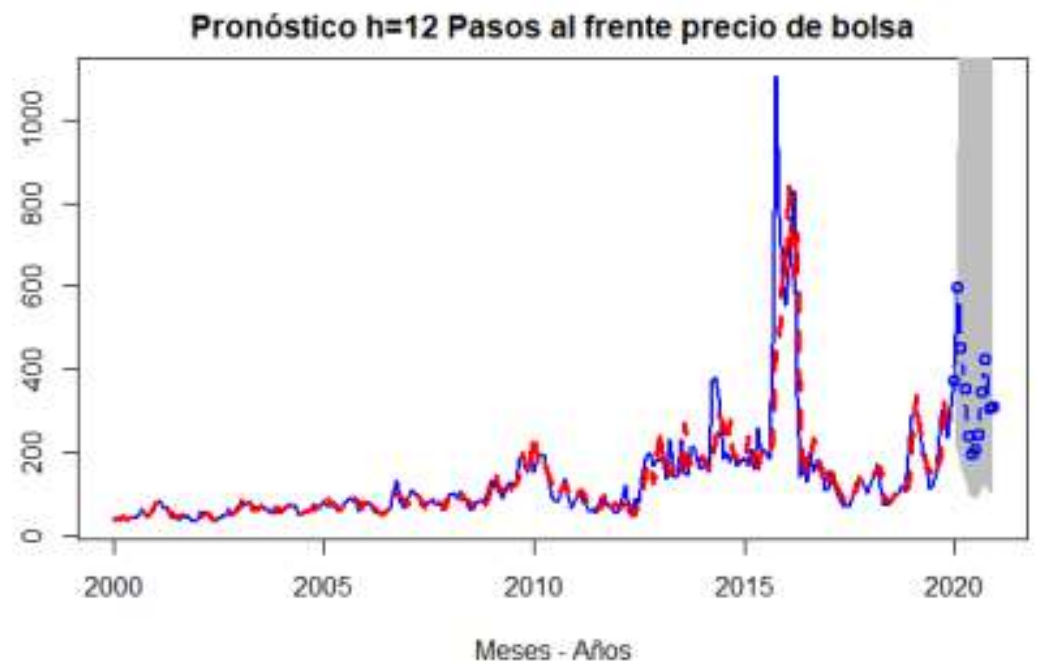

Fig. 8. Ajuste y pronóstico Modelo SARIMA sobre comportamiento del Precio de Bolsa. Valores en $\$ / K W h$.

En la Fig. 9. Ajuste Modelo SARIMA a precios observados en 2020, se comparan los valores pronosticados por el Modelo 1 (línea roja) para el año 2020 con los valores realmente registrados durante este periodo (línea azul), respecto a las bandas de confianza establecidas. Tal como se puede observar, se puede afirmar que los valores pronosticados se acercan de manera aceptable a los valores reales.

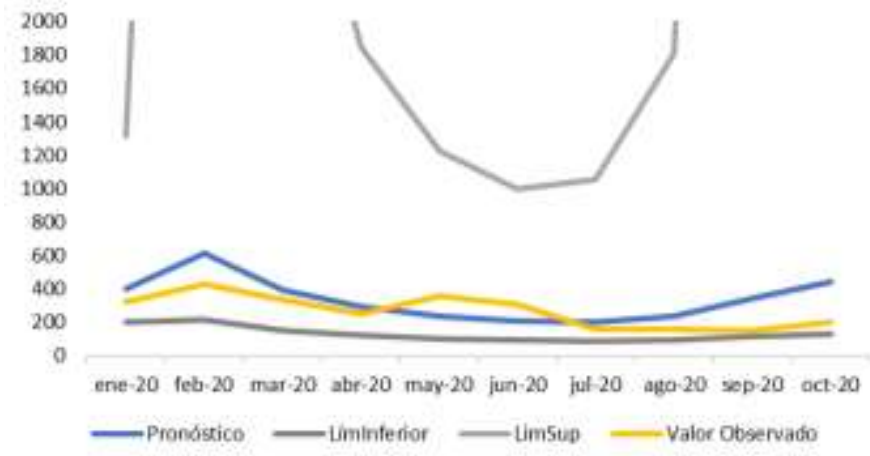

Fig. 9. Ajuste Modelo SARIMA a precios observados en 2020. Valores en \$/KWh.

\section{Evaluación y pronóstico modelo SARIMAX}

Bajo la metodología SARIMAX se evaluaron 5 modelos, de los cuales se eligió el modelo descrito en la Tabla 3, para el cual se utilizaron como variables exógenas los aportes y el volumen útil del sistema, rezagadas en 6 periodos. 
Tabla 3. Pruebas Modelo SARIMAX.

\begin{tabular}{|c|l|c|r|r|r|}
\hline Nombre & \multicolumn{1}{|c|}{ Descripción } & BIC & Box.test & jarque.bera & runs.test \\
\hline Modelo 2 & $\begin{array}{l}\text { SARIMAX }(0,1,10)(0,1,1)[12] \\
\text { Variables exógenas: Aportes y } \\
\begin{array}{l}\text { Volumen. } \\
\text { Rezagos: } 6\end{array}\end{array}$ & 2601.593 & 0,9573 & 0 & 0.7546 \\
\hline
\end{tabular}

Al igual que el modelo SARIMA, este modelo tampoco tiene explicación en el componente autorregresivo estacional y ordinario, por lo tanto, la ecuación puede expresarse como:

$$
\begin{aligned}
& \nabla_{S}^{D} \nabla^{d} x_{t}=\Theta_{Q}\left(B^{S}\right) \theta(B) w_{t}+\sum_{k=1}^{r} B y_{t-k} \text { donde } \\
& \nabla_{S}^{D}=\left(1+B^{12}\right) \\
& \nabla^{d}=(1+B) \\
& \theta_{q}=\left(1-0,3427 w_{t-2}-0,1414 w_{t-4}-0,3053 w_{t-5}-0,4077 w_{t-6}-0,3641 w_{t-7}-0,1645 w_{t-9}\right. \\
& \left.\quad-0,2098_{t-10}\right) \\
& \Theta_{Q}=\left(1-0,9196 w_{t-12}\right) \\
& \sum_{k=1}^{r} B y_{t-k}=0,0009 y_{t-6}-2,1331 z_{t-6} \\
& x_{t}=\text { Precio de bolsa en el periodo } t \\
& w_{t}=\text { Ruido blanco en el periodo } t \\
& y_{t}=\text { Valor de los aportes en el periodo } \mathrm{t} \\
& z_{t}=\text { Valor del volumen útil del embalse agregado en el periodo } \mathrm{t} \\
& k=\text { Rezagos }
\end{aligned}
$$

El comportamiento del pronóstico (línea azul) sobre el precio de bolsa parece ajustarse a los datos hasta el año 2013 a partir de este período tiene desfases y retoma el comportamiento ajustado entre el 2018 y 2020, así mismo la proyección de seis períodos en el futuro se encuentra dentro de las bandas de confianza como se puede ver en la Fig. 10. 


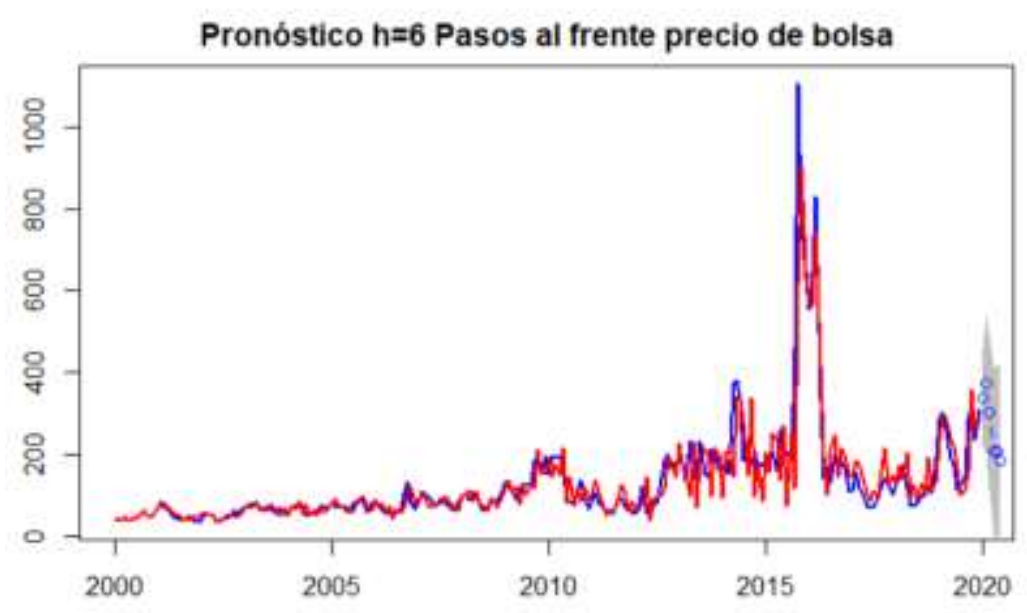

Fig. 10. Ajuste y pronóstico Modelo SARIMAX sobre comportamiento del Precio de Bolsa. Valores en $\$ / K W h$.

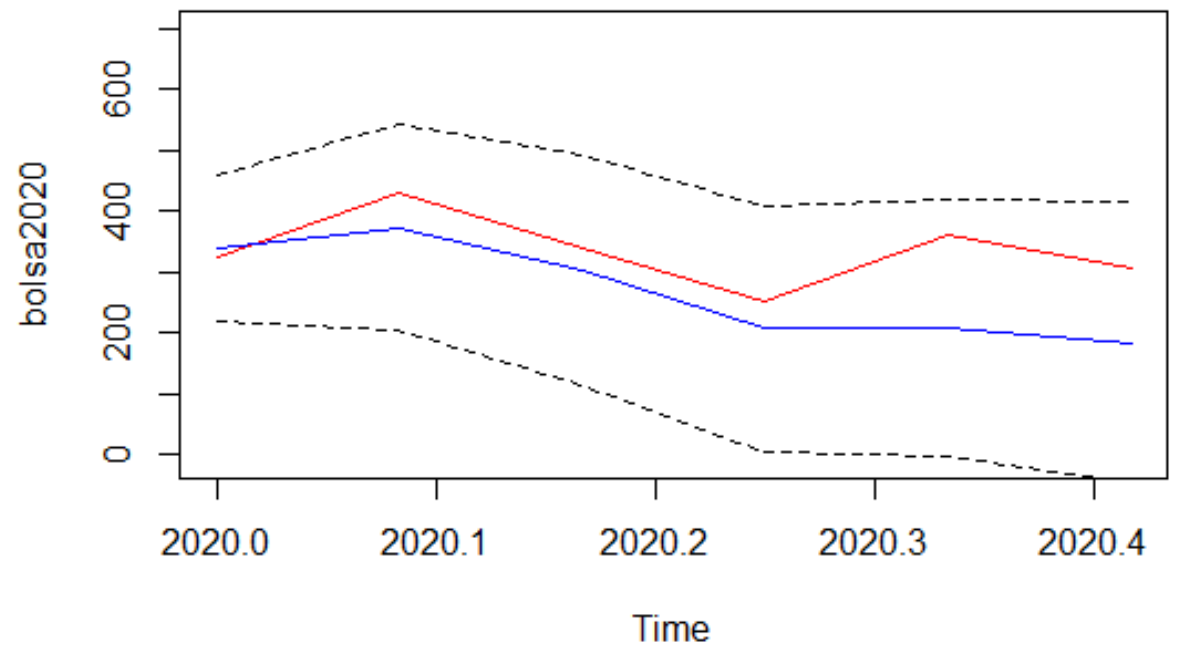

Fig. 11. Ajuste del Modelo SARIMAX sobre comportamiento del Precio de Bolsa en el 2020. Valores en $\$ / \mathrm{KWh}$.

El comportamiento detallado de la proyección de los datos en series períodos permite observar que el valor real de los precios de bolsa (línea roja) y la proyección se encuentran dentro de las bandas de confianza y que el pronóstico tiene un ajuste cercano a los valores reales. 


\section{Evaluación y pronóstico modelo VAR}

Bajo el análisis de modelo VAR se evaluaron 14 modelos para explicar los precios de bolsa, de los cuales el modelo elegido es el descrito en la Tabla 4, el cual tiene como única variable explicativa a la serie disponibilidad con 2 rezagos. En esa misma tabla se presentan las pruebas de evaluación, a partir de las cuales se encontró que el modelo no pasa las pruebas de correlación de Portmanteau, heteroscedasticidad de $\mathrm{ARCH}$, ni la prueba de normalidad de los residuales.

Tabla 4. Pruebas Modelo VAR.

\begin{tabular}{|l|l|r|r|r|r|}
\hline Nombre & \multicolumn{1}{|c|}{ Descripción } & BIC & Portmanteau & ARCH & \multicolumn{1}{|c|}{$\begin{array}{c}\text { Test de } \\
\text { normalidad }\end{array}$} \\
\hline Modelo 5 & $\begin{array}{l}\text { VAR } \\
\begin{array}{l}\text { Variable } \\
\text { disponibilidad } \\
\text { Rezagos=2 }\end{array}\end{array} \quad$ exógena: & 9762.22 & 0.0001631 & 0.1320 & $2.2 \mathrm{e}-16$ \\
\hline
\end{tabular}

Este modelo se encuentra representado por la siguiente ecuación:

$x_{t}=0,0000018-0,0897 x_{t-1}+0,494 y_{t-1}+0,00172 x_{t-2} 0,344 y_{t-2}$

Donde

$x_{t}=$ Precio de bolsa en el periodo $t$

$y_{t}=$ Valor de la disponibilidad del parque generador en el periodo $\mathrm{t}$

Sin embargo, este modelo fue seleccionado entre los modelos VAR debido a que presenta el mejor ajuste gráfico entre los valores de precio de bolsa generados por el modelo y los valores de precio de bolsa observados.

El pronóstico del modelo elegido parece acomodarse a la serie de tiempo (línea roja), al seguir los picos y valles de la serie. Cabe señalar, que el ajuste del modelo presenta unos desfases con relación a los valores observados y que en algunos momentos el modelo se ajustó a los datos observados, como se observa a finales del año 2014 y a inicios del año 2014. Adicionalmente, se puede afirmar que, bajo este modelo, para el año 2020 se espera una disminución de los precios de bolsa (Fig. 12). 


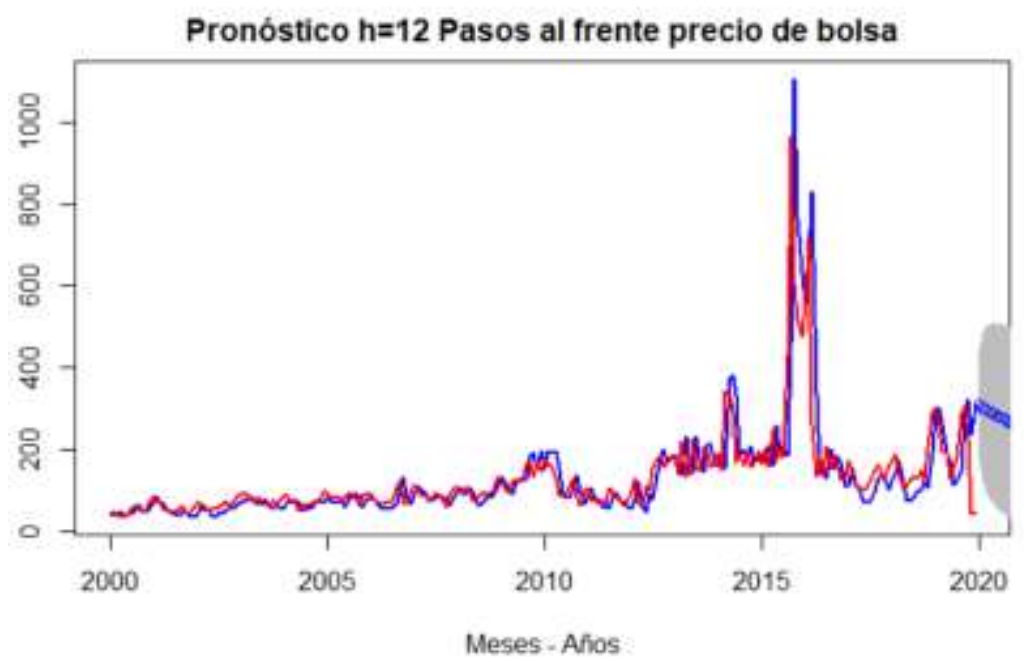

Fig. 12. Ajuste y pronóstico Modelo VAR sobre comportamiento del Precio de Bolsa. Valores en $\$ / K W h$.

En la Fig. 13, se comparan los valores pronosticados por el Modelo 5 para el año 2020 con los valores realmente registrados durante este periodo, respecto a las bandas de confianza establecidas. Tal como se puede observar, se puede afirmar que los valores pronosticados se acercan de manera aceptable a los valores reales y se encuentran al interior de las bandas de confianza.

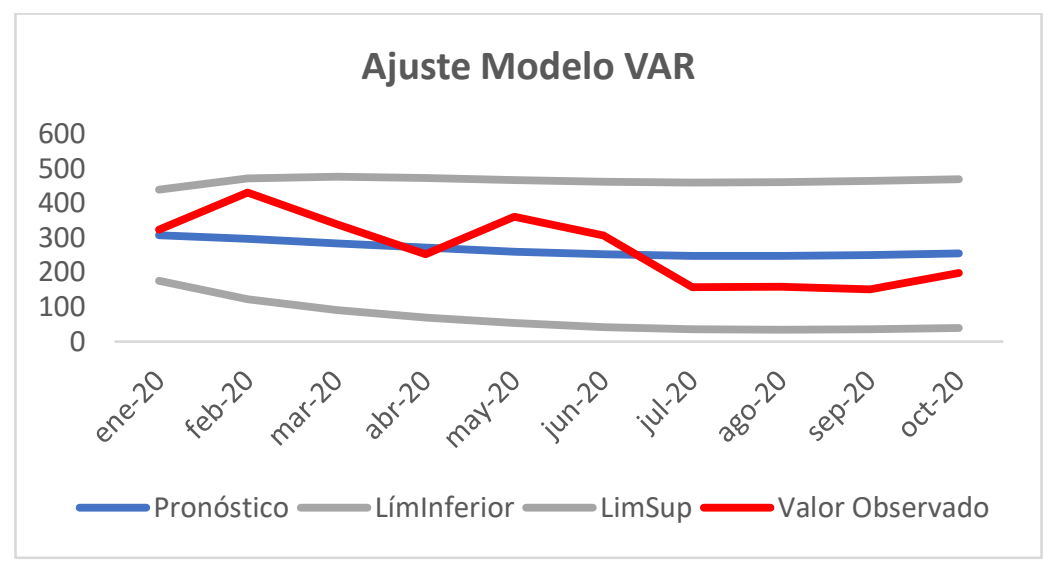

Fig. 13. Ajuste Modelo VAR a precios observados en 2020. Valores en $\$ / K W h$.

\section{Selección del mejor modelo}

Para seleccionar el mejor modelo se realiza la prueba del Error Cuadrático Medio (RSME) para medir la cantidad de error que hay entre dos conjuntos de datos, es 
decir, para comparar el valor pronosticado por cada modelo con el valor observado o conocido.

$R S M E=\sqrt{\frac{\sum_{i=1}^{n}\left(P_{i-} O_{i}\right)^{2}}{n}}$

Donde:

$\mathrm{n}=$ Número de periodos de la serie de tiempo

$\mathrm{P} \mathrm{i}=$ Valor pronosticado periodo $\mathrm{i}$

$\mathrm{O}_{\mathrm{i}}=$ Valor observado en el periodo $\mathrm{i}$

Para calcular el RSME, en la Tabla 5 se observan los valores observados para el precio de la energía en bolsa durante el 2020, frente a los valores pronosticados bajo los modelos evaluados según SARIMAX, SARIMA y VAR.

Tabla 5. Comparación precios observados en 2020 frente a los pronosticados bajo los modelos evaluados. Valores en $\$ / K W h$.

\begin{tabular}{|r|l|r|r|r|r|}
\hline \multirow{2}{*}{ Año } & \multirow{2}{*}{ Mes } & \multirow{2}{*}{$\begin{array}{c}\text { Valor } \\
\text { observado }\end{array}$} & \multicolumn{3}{|c|}{ Valor pronosticado } \\
\cline { 4 - 6 } & & 323,1 & 337,7 & 402,0 & 307,1 \\
\hline 2020 & Enero & 430,5 & 371,9 & 610,5 & 296,9 \\
\hline 2020 & Febrero & 339,5 & 302,4 & 395,4 & 283,4 \\
\hline 2020 & Marzo & 252,2 & 206,2 & 296,9 & 271,4 \\
\hline 2020 & Abril & 361,0 & 208,9 & 239,9 & 260,0 \\
\hline 2020 & Mayo & 306,5 & 183,3 & 207,5 & 252,1 \\
\hline 2020 & Junio & 156,8 & 208,5 & 202,8 & 247,8 \\
\hline 2020 & Julio & 159,0 & 214,1 & 235,6 & 247,5 \\
\hline 2020 & Agosto & 150,9 & 237,4 & 343,8 & 250,0 \\
\hline 2020 & Septiembre & 198,1 & 299,6 & 440,2 & 254,4 \\
\hline 2020 & Octubre & & & & \\
\hline
\end{tabular}

En la Tabla 6, se relacionan los resultados del cálculo del Error Cuadrático Medio para cada uno de los modelos evaluados, según los cuales el VAR y SARIMAX tienen los valores más bajos, frente al modelo SARIMA que supera ampliamente los valores de los dos primeros. Bajo este resultado se elige el modelo SARIMAX, debido a que el modelo VAR no pasa las pruebas de correlación y heterocedasticidad. 
Tabla 6. Resultado Error Cuadrático Medio para cada uno de los modelos

\begin{tabular}{|c|c|l|}
\hline \multicolumn{3}{|c|}{ RSME } \\
\hline SARIMAX & SARIMA & VAR \\
\hline $83-0$ & 131.2 & \\
\hline
\end{tabular}

\section{Conclusiones}

Desde el punto de vista estadístico se identificaron los tres mejores modelos SARIMAX, SARIMA y VAR, los cuales fueron comparados en términos de bondad de ajuste y en términos de las pruebas que fueron superadas para cada uno. A pesar de que los tres modelos lograron ubicar el pronóstico dentro de las bandas de confianza, se eligió el modelo SARIMAX porque pasa las pruebas de correlación y heterocedasticidad, además presentó una prueba de bondad de ajuste aceptable.

Según los resultados pronosticados por este modelo, los precios de bolsa en el 2020 sufrirían un descenso respecto a lo sucedido a finales del 2019; esta tendencia fue confirmada en parte por los valores observados realmente hasta abril, sin embargo, a partir de mayo se presentan diferencias importantes entre lo pronosticado por el modelo y la realidad.

A partir de estos resultados, se puede identificar que si bien, el modelo permite predecir de una manera aceptable el comportamiento del precio de energía en bolsa en Colombia, sería importante incluir otras metodologías como redes neuronales o aprendizaje de máquinas para mejorar el ajuste de los pronósticos.

Así, mismo para el modelo VAR, el cual no pasó las pruebas de heterocedasticidad y correlación, se recomienda mejorarlo por medio de un modelo VARMA que contemple el componente de medias móviles tanto de la variable precio de bolsa como de las variables explicativas.

\section{Referencias}

Alvarez, S., y Tamayo, M. P. (2006). Descripción del funcionamiento del sector eléctrico colombiano. Ecos de Economìa, 10(22), 7-44

Barrero Garcia, J. E. (2019). Educación ambiental para la paz Una mirada desde la formación académica en Suboficiales del Ejército Nacional de Colombia. Noria Investigación Educativa, 2(4), 5-10. https://doi.org/10.14483/25905791.16328

Barrientos, J., y Toro, M. (2016). La hidrología como predictor del comportamiento del precio de energía en 
bolsa. Perfil de Coyuntura Económica, 25, e7. https://doi.org/10.17533/udea.pece.n25a07

Bello, S., y Beltrán, R. (2010). Caracterización y pronóstico del precio spot de la energía eléctrica en Colombia. Revista de La Maestría En Derecho Económico, 6, 293-316

República de Colombia. (1994). Ley 142 - Por la cual se establece el régimen de los servicios públicos domiciliarios y se dictan otras disposiciones

República de Colombia. (1994). Ley 143 - Por la cual se establece el régimen para la generación, interconexión, transmisión, distribución y comercialización de electricidad en el territorio nacional, se conceden unas autorizaciones y se dictan otras disposiciones en materia energética

Cruz Hurtado, E. J., y Romero, M. F. (2020). Propuesta de un modelo logístico para la probabilidad de instalación de datáfonos en una empresa ubicada en Bogotá. Noria Investigación Educativa, 1(5), 41-53. https://doi.org/10.14483/25905791.16452

Díez, I. C. (2015). Proyección de precios de energía eléctrica de mediano plazo en el mercado colombiano mediante la aplicación del índice de Lerner [Trabajo de grado]. Universidad EAFIT, Medellín, Colombia

Galindo, A. F. (2017). Modelamiento de los precios de la energía en bolsa en Colombia incorporando el efecto del ENSO [Trabajo de grado]. Universidad de Los Andes, Bogotá D.C., Colombia

Gil, M., y Maya, C. (2008). Modelación de la volatilidad de los precios de la energía eléctrica en Colombia. Revista de Ingenierías: Universidad de Medellín, 7(12), 87-114

Hurtado, L., Quintero, O. L., y García, J. J. (2014). Estimación del precio de oferta de la energía eléctrica en Colombia mediante inteligencia artificial. Revista de Métodos Cuantitativos Para La Economía y La Empresa, 18, 54-87

Martın, R. A., y Pineros, L. F. (2020). Propuesta de un modelo con redes neuronales y metodología Box \& Jenkins para el pronóstico del precio de bolsa de la energía en Colombia [Trabajo de grado]. Fundación Universitaria Los Libertadores, Bogotá D.C., Colombia

Porras Contreras, Y. A. y Pérez Mesa, M. R. (2019). Identidad ambiental: múltiples perspectivas. Revista científica, (34), 123-138. https://doi.org/10.14483/23448350.14003

Rendón, J. G., Hinestroza, A. G., \& Moreno, L. S. (2011). Determinantes del precio de la energía eléctrica en el mercado no regulado en Colombia. Revista Ciencias Estratégicas, 19(26), 225-246.

Rojas, C. F. (2016). Imperfecciones en el mercado eléctrico colombiano y comportamientos estratégicos de los agentes Un análisis desde la teoría de juegos para el mercado spot. 1-38. https://core.ac.uk/download/pdf/84841888.pdf

Sabogal Florido, S. I. (2016). Evaluación del efecto de las variables: generación, aportes y demanda de energía del sistema interconectado nacional en el precio de bolsa nacional de energía en Colombia periodo (20002014).

Salamanca Céspedes, J. E. (2019). La educación en energías renovables no convencionales en la formación de ingenieros electrónicos. Noria Investigación Educativa, 2(4), 11-18. https://doi.org/10.14483/25905791.16331

Santamaría, M., Feh Von der, N.-H., Millán, J., Benavides, J., Gracia, O., y Schutt, E. (2009). El mercado de la energía eléctrica en Colombia características, evolución e impacto sobre otros sectores

XM. (2019). Gestión de los mercados con múltiples energéticos. https://www.energycolombia.org/wpcontent/uploads/Gestiondelosmercados XM.pdf

XM. (2020). Portal BI XM. http://portalbissrs.xm.com.co/Paginas/Home.aspx 